\title{
LAMBDA BOO ABUNDANCE PATTERNS: ACCRETION FROM ORBITING SOURCES
}

\author{
M. Jura ${ }^{a}$
}

\begin{abstract}
The abundance anomalies in $\lambda$ Boo stars are popularly explained by elementspecific mass inflows at rates that are much greater than empirically-inferred bounds for interstellar accretion. Therefore, a $\lambda$ Boo star's thin outer envelope must derive from a companion star, planet, analogs to Kuiper Belt Objects or a circumstellar disk. Because radiation pressure on gas-phase ions might selectively allow the accretion of carbon, nitrogen, and oxygen and inhibit the inflow of elements such as iron, the source of the acquired matter need not contain dust. We propose that at least some $\lambda$ Boo stars accrete from the winds of hot Jupiters.
\end{abstract}

Subject headings: planetary systems - stars, main-sequence

\section{INTRODUCTION}

Approximately $2 \%$ of main sequence A-type stars are similar to $\lambda$ Boo in having essentially solar carbon, nitrogen and oxygen abundances but subsolar abundances of heavier elements such as iron (Paunzen 1991; Gray \& Corbally 1992). A plausible model to explain these distinctive abundances is that these stars have accreted gas but not dust (Venn \& Lambert 1990). In this scenario, radiation pressure from the star prevents grains with their high opacity from accreting while gas is not so inhibited (Kamp \& Paunzen 2002; Martinez-Galarza et al. 2009).

Although the general picture that $\lambda$ Boo stars acquired their distinctive abundances by selective accretion is promising, there are objections which must be resolved (Cowlev 2014). For example, most $\lambda$ Boo stars do not exhibit an infrared excess (King 1994; Paunzen et al. 2003). Consequently, there is no evidence for either circumstellar dust or nearby interstellar dust. Also, main-sequence A-type stars with dust do not always exhibit $\lambda$ Boo photospheric

\footnotetext{
a Department of Physics and Astronomy, University of California, Los Angeles CA 90095-1562; jura@astro.ucla.edu
} 
abundances (Kamp et al. 2002). Seemingly, unless there is undetected dust, some additional mechanism to explain selective elemental accretion should be considered.

The popular model for $\lambda$ Boo stars requires a substantial accretion rate. In particular, depending upon the amount of deep mixing and meridional circulation, required accretion rates range between $6 \times 10^{11} \mathrm{~g} \mathrm{~s}^{-1}$ and $6 \times 10^{14} \mathrm{~g} \mathrm{~s}^{-1}$ (Turcotte \& Charbonneau 1993; Turcotte 2002), comparable to the solar wind outflow of $\sim 10^{12} \mathrm{~g} \mathrm{~s}^{-1}$. As described in detail below, these values are orders of magnitude greater than empirically-determined bounds on accretion rates by stars from the interstellar medium. Therefore, the thin outer envelope must be acquired from an orbiting source close to the star.

A process that has been invoked to explain the anomalous circumstellar abundances of $\beta$ Pic (Fernandez et al. 2006; Xie et al. 2013) but not yet considered for $\lambda$ Boo stars is radiation pressure on individual ions. This effect is highly element specific and therefore may be important in explaining the abundance pattern in $\lambda$ Boo stars. If so, then dust need not be a constituent in the reservoir of the accreted material, allowing us to consider sources for accretion onto $\lambda$ Boo stars that were previously neglected.

In Section 2, we examine and discard the possibility of interstellar accretion. In Section 3 , we assess the possibility that radiation pressure on atoms and ions can act to allow for selective accretion with the characteristic pattern seen in $\lambda$ Boo stars. In section 4 , we assess various possible sources of the matter accreted onto $\lambda$ Boo stars and suggest that one unappreciated possibility is the wind from an irradiated hot Jupiter. We discuss our results in Section 5 and our conclusions in Section 6.

\section{INTERSTELLAR ACCRETION?}

Martinez-Galarza et al. (2009) used Bondi-Hoyle theory to compute interstellar accretion onto $\lambda$ Boo stars. Because the applicability of this approach is uncertain, Koester 1976; Farihi et al. 2010), empirical measures of accretion rates should be considered. A sensitive upper bound on the time-averaged rates of interstellar accretion onto stars can be derived from the amount of hydrogen found in the outer mixing zones of DB white dwarfs, stars where the dominant element in the atmosphere is helium. Because much or all of this hydrogen either had a circumstellar origin or was primordial (Bergeron et al. 2011), only upper bounds can be derived for the rate of interstellar accretion, $d M / d t$. In a catalog of 57 DBs within $80 \mathrm{pc}$ of the Sun, the time-averaged value of $d M / d t$ typically is less than or equal to $10^{6} \mathrm{~g} \mathrm{~s}^{-1}$ (Jura \& Xu 2012).

For stars of mass, $M_{*}$, the Bondi-Hoyle accretion rate varies as $M_{*}^{+2}$. White dwarf 
stars typically have 1/3 the mass of a $\lambda$ Boo star (Kleinman et al. 2013; Paunzen et al. 2002), and therefore their accretion rates from the interstellar medium might be a factor of 10 lower. However, even making this adjustment, the time-averaged accretion rate inferred for DB white dwarfs is nearly a factor of $10^{5}$ lower than the minimum required rate. Because $\sim 50 \%$ of the $34 \lambda$ Boo stars discussed by Heiter et al. (2002) lie within 80 pc of the Sun and therefore within the local interstellar bubble (Lallement et al. 2003), this comparison with nearby white dwarfs is meaningful.

We also consider empirical measures of "instantaneous" accretion. We therefore use measures of accretion rates onto DA white dwarfs (stars where the atmosphere is dominated by hydrogen) that are warmer than $13,000 \mathrm{~K}$ where the settling time of heavy elements below the photosphere is only days (Koester 2009). In a sample of 87 such stars, the maximum carbon accretion rate is $2 \times 10^{6} \mathrm{~g} \mathrm{~s}^{-1}$ (Koester 2009). Because solar abundances (Lodders $2003)$ are assumed for the source material, $\lambda$ Boo stars are required to accrete carbon at a rate of $2 \times 10^{9} \mathrm{~g}^{-1}$, a factor of 1000 greater than the maximum value measured for white dwarfs. The true discrepancy is probably substantially larger because the most likely source of the white dwarf's photospheric carbon is circumstellar rather than interstellar (Jura \& Young 2014). We conclude that empirical bounds on interstellar accretion rates are much lower than what is required to explain $\lambda$ Boo stars.

\section{ACCRETION GOVERNED BY RADIATION PRESSURE}

Before exploring the different possible sources of accretion onto $\lambda$ Boo stars, we consider an additional element-sorting mechanism for sources that are dust-free. Specifically, we extend models invoking radiation pressure on individual ions developed to explain the distinctive composition of $\beta$ Pic's circumstellar gas to $\lambda$ Boo stars.

Following notational convention, we define $\beta$ as the ratio of the outward force of radiation pressure compared to the inner force of gravity. If the star has radius, $R_{*}$, effective temperature, $T_{*}$, mass, $M_{*}$ and emergent flux at the stellar surface, $F_{\nu}$, then:

$$
\beta=\left(\frac{\pi e^{2}}{m_{e} c^{2}} \sum_{j} f_{j} F_{\nu}\right)\left(g m_{i}\right)^{-1} .
$$

Here, $e$ denotes the charge of an electron of mass, $m_{e}, c$ is the speed of light, $g$ is the gravitational acceleration at the photosphere and $m_{i}$ is the mass of the $i$ 'th ion with resonance transitions of oscillator strength, $f_{j}$. We consider environments sufficiently far from the host star that ions mostly lie in their ground states.

Qualitatively, using the Wien approximation to the Planck curve and assuming that in 
the ultraviolet the opacity is so high that we observe only the upper layers of the atmosphere and that the photospheric lines are weak, we write that:

$$
F_{\nu} \sim \frac{2 \pi h \nu^{3}}{c^{2}} e^{-h \nu / k T_{\min }}
$$

where $T_{\min }$ is the temperature minimum in the photosphere or $\sim 0.75 T_{*}$ (Kurucz 1979). A low value of $\beta$ requires a low value of $F_{\nu}$ which requires that the resonance lines lie at relatively short wavelengths. Important lines for C II , N I and O I lie at $1335 \AA, 1200 \AA$ and $1302 \AA$, respectively, while, in contrast, important lines for $\mathrm{Mg}$, Ca and Fe lie at $2802 \AA, 3933$ $\AA$ and $2599 \AA$, respectively (Morton 1991). Therefore, C, N and O can be accreted while $\mathrm{Mg}, \mathrm{Ca}$ and $\mathrm{Fe}$ cannot. This qualitative expectation is borne out by detailed calculations (Fernandez et al. 2006) for a model of $\beta$ Pic with assumed values of $T_{*}=8000 \mathrm{~K}$ and $\log g$ $=4.2$, stellar parameters appropriate for many $\lambda$ Boo stars (Heiter et al. 2002). We show in Figure 1 a plot for $\lambda$ Boo of an element's abundance, compared to its solar abundance, $X$, vs. the relative importance of outward radiation pressure, $\beta$, where:

$$
X=\log \left(\frac{n(X)}{n(H)}\right)_{*}-\log \left(\frac{n(X)}{n(H)}\right)_{\odot}
$$

There are two classes of elements: those with high values of $X$ and low values of $\beta$ in their likely dominant state of ionization and those with low values of $X$ and high values of $\beta$.

This analysis extend to elements beyond those considered in Figure 1. Both $\mathrm{H}$ and $\mathrm{S}$ also are computed to have low values of $\beta$ and can be accreted while $\mathrm{Si}$ and Mn have high values of $\beta$ (Fernandez et al. 2006) and would be inhibited from accreting, consistent with the general pattern of abundances among $\lambda$ Boo stars (Heiter 2002).

One complication to this picture is that there are some elements, such as $\mathrm{Na}$, where $\beta$ can be high in one state of ionization and low in another. For such an element, the net balance among all the potential states must be determined in order to assess whether it is accreted. Although there are uncertainties, we argue that outward radiation pressure on individual elements might play a key role in explaining the abundance pattern of $\lambda$ Boo stars.

\section{ORBITING SOURCES}

The alternative to interstellar accretion is accretion from some material gravitationally bound to the host star. We now assess different possibilities. 


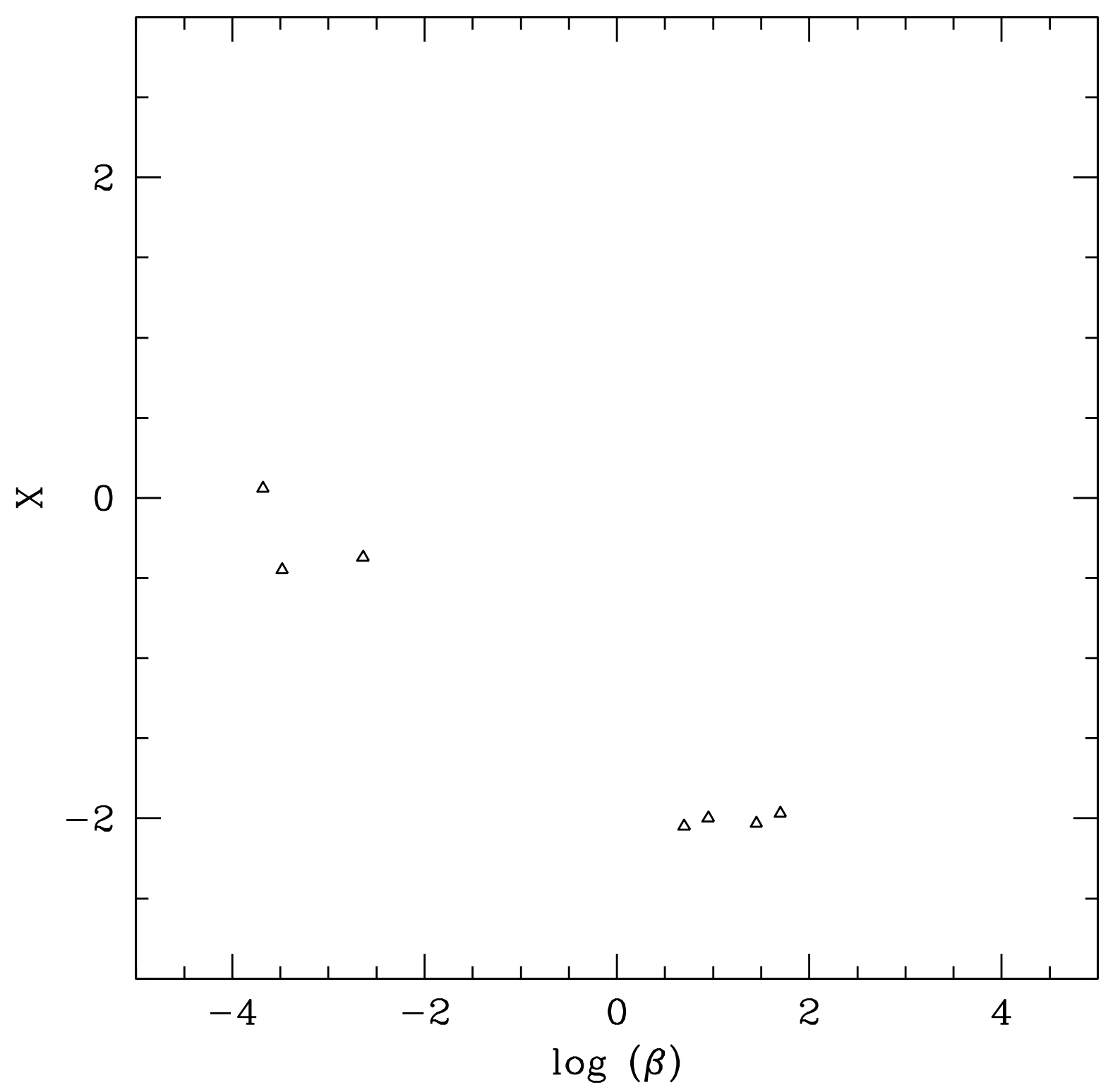

Fig. 1.- Plot of abundance relative to solar (Venn \& Lambert 1990) defined by $X$ in Equation (3) vs. $\beta$ defined in Equation (1) for different elements in their likely dominant state of ionization (Fernandez et al. 2006): C II, N I, O I, Fe II, Ca II, Ti II, Mg II. The three elements with approximately solar abundances are $\mathrm{C}, \mathrm{N}$ and $\mathrm{O}$. A clear distinction in values of $X$ between those with high $\beta$ and those with low $\beta$ is evident. 


\subsection{Companion Stars?}

A possible scenario to explain $\lambda$ Boo abundances is the accretion of the outflow from a stellar companion. In fact, there are rare instances of spectroscopic binary systems where two stars orbit close to each other and one star exhibits a $\lambda$ Boo abundance pattern (Narusawa et al. 2006; Waters et al. 1992). However, such situations probably do not explain most $\lambda$ Boo stars (Stutz \& Paunzen 2006; Griffin et al. 2012). A binary configuration of two more distant stars which might account for some $\lambda$ Boo objects would be an A-type star accreting from the wind of a late type companion. The typical mass loss rate from a late-type main-sequence star is $\sim 10^{12} \mathrm{~g} \mathrm{~s}^{-1}$ (Wood et al. 2002), comparable to the mass accretion rate required to explain $\lambda$ Boo stars. Because the characteristic wind speeds from late type stars likely are near their escape speed of $600 \mathrm{~km} \mathrm{~s}^{-1}$, only a small fraction of the matter lost from the companion can be accreted by the $\lambda$ Boo star (Debes 2006). Therefore, accretion of winds from late-type stellar companions seems unlikely to explain most $\lambda$ Boo stars.

\subsection{Rocky Planets or Asteroids?}

The carbon to oxygen ratio measured in the atmospheres of $\lambda$ Boo stars typically is within a factor of three of the solar ratio (Heiter et al. 2002). In contrast, most matter from rocky minor planets accreted onto white dwarfs has a carbon to oxygen ratio that is typically a factor of 100 lower than solar (Jura \& Young 2014). This marked deficiency of carbon within extrasolar asteroids - the building blocks of rocky planets - argues against their being a major reservoir of matter accreted onto $\lambda$ Boo stars.

\subsection{Kuiper Belt Analogs or Comets?}

Another source of accreted material might be analogs to Kuiper Belt Objects or comets with carbon to oxygen abundance ratios that might be nearly solar (Jura et al. 2015) although perhaps not (Wilson et al. 2015). Zuckerman \& Song (2012) have proposed that colliding comets explain the observed gas around 49 Cet, a young main sequence A-type star. The suggested CO production rate is greater than $\times 10^{13} \mathrm{~g} \mathrm{~s}^{-1}$, large enough to induce $\lambda$ Boo abundances. However, there are difficulties with this model. It is not clear how matter initially at $\geq 100$ AU ultimately accretes onto the host star. Also, comets by consisting largely of water, may have very subsolar hydrogen to oxygen ratios. Accretion of such material might produce an oxygen overabundance which is not observed. Finally, 49 Ceti has an 
infrared excess while most $\lambda$ Boo stars do not. Given these objections, alternative reservoirs for the accretion onto $\lambda$ Boo stars should be considered.

\subsection{Pre-Main-Sequence Disks?}

In the absence of accretion, mixing erases the abundance anomalies in $\sim 10^{6}$ yr (Turcotte \& Charbonnea 1993). While accretion from a primordial disk may lead to the $\lambda$ Boo abundance pattern measured in numerous pre-main-sequence stars (Folsom et al. 2014; Cowley et al. 2014), there are many $\lambda$ Boo stars that are sufficiently old that their photospheric abundances are not a relic from the era of their formation (Paunzen et al. 2002). A recent source of accretion should be considered.

\subsection{Hot Jupiters?}

Because the source must be substantial and likely located relatively near the star to guarantee ultimate accretion, we propose that the source of matter accreted onto many $\lambda$ Boo stars is the wind from a close-in planet. As an example, consider WASP 33 (or HD 15082), an A5 main-sequence star orbited by a hot Jupiter with a period of $1.22 \mathrm{~d}$ (Collier-Cameron et al. 2010). The mass loss rate from this planet may be $2 \times 10^{13} \mathrm{~g} \mathrm{~s}^{-1}$ (Bourrier et al. 2015), sufficiently high to explain $\lambda$ Boo stars.

Here, we assume that all the mass lost from the planet ultimately is acquired by the star. Furthermore, we also assume that individual ions can separate from the general flow as supported by a toy model not presented here. Detailed calculations are required to assess these requirements. For example, in the case of the outer circumstellar gas orbiting $\beta$ Pic, the effectiveness of the separation between different elements depends upon the viscosity in the gas (Xie et al. 2013).

In models where the upper atmosphere is heated by ultraviolet and X-ray photons, the wind rate, $\dot{M}$, from a planet at distance $D$ from the can star can be estimated as:

$$
\dot{M}=\frac{3 \eta}{16 \pi G \rho_{p}} \frac{L_{E U V}}{D^{2}},
$$

where $G$ is the gravitational constant, $L_{E U V}$ is the total stellar EUV luminosity in the spectral range $\lambda<912 \AA, \rho_{p}$ is the planet's mean density, and $\eta$ is an efficiency factor to describe the conversion of incident radiative energy to kinetic energy in the outflowing wind (Murray-Clav et al. 2009). This formula may overestimate the planetary mass loss rate if the planet's magnetic field is sufficiently large (Owen \& Adams 2014). 
Now consider "optimistic" values of the different parameters in Equation (4). We take $R_{p}$ of and $\rho_{p}$ for WASP $17 \mathrm{~b}$ of $1.3 \times 10^{11} \mathrm{~cm}$ and $0.062 \mathrm{~g} \mathrm{~cm}^{-3}$, respectively (Anderson et al. 2011), the most inflated hot Jupiter currently known (Bento et al. 2014). We assume $D$ $=6.0 \times 10^{11} \mathrm{~cm}$ in order for the planet to be stable against tidal disruption (Paczynski 1971). We also take $\eta=1$. Because of attenuation in the interstellar medium, the EUV luminosity of most stars is unobservable and therefore highly uncertain. Indirect estimates of $L_{U V}$ can be derived from observed subcoronal emission lines characteristic of gas with temperatures between 50,000 K and 300,000 K. Such lines have been detected in stars with $T<8200 \mathrm{~K}$ although stars as hot as $8800 \mathrm{~K}$ may possess coronae (Simon et al. 2002). Further, approximately 10-15\% of main sequence A-type stars are detected X-ray sources; the underlying physical explanation is not known (Schroder \& Schmitt 2007). Here, to be specific, we consider for comparison the well studied star, $\beta$ Pic, with $T=8200 \mathrm{~K} \pm 150$ $\mathrm{K}$ (Lanz et al. 1995), a representative temperature for many $\lambda$ Boo stars. For $\beta$ Pic, the measured X-ray luminosity between 0.2 and $20 \mathrm{keV}$ is $3 \times 10^{26} \mathrm{erg} \mathrm{s}^{-1}$, and the extrapolated $\mathrm{X}$-ray luminosity between 0.06 and $5 \mathrm{keV}$ is $3 \times 10^{27} \mathrm{erg} \mathrm{s}^{-1}$ (Gunther et al. 2012). There must also be emitted photons with energies between 0.0136 and $0.06 \mathrm{keV}$. According to Bouret et al. (2002), the total chromospheric emission of $\beta$ Pic is at least $9.9 \times 10^{28} \mathrm{erg}$ $\mathrm{s}^{-1}$. While much of this energy is emitted with $\lambda>912 \AA$, an appreciable fraction is thought to be emitted at $\lambda<912 \AA$. Here, recognizing that there is great uncertainty, we adopt $L_{E U V}$ $=2 \times 10^{28} \mathrm{erg} \mathrm{s}^{-1}$ or $L_{X} / \mathrm{L}_{b o l} \sim 5 \times 10^{-7}$. If so, then $\dot{M} \approx 10^{12} \mathrm{~g} \mathrm{~s}^{-1}$, sufficiently large to explain $\lambda$ Boo abundances.

One complication is that a hot Jupiter may have a sufficiently short period that it can induce activity on its host star. Shkolnik et al. (2005) have reported evidence to support $\sim 10^{27} \mathrm{erg} \mathrm{s}^{-1}$ of such dissipation. Accordingly, through magnetically controlled interactions, the host planet might lose $10^{12} \mathrm{~g} \mathrm{~s}^{-1}$ (Lanza 2013). However, it is uncertain whether models developed for later type stars apply to A-type stars, but if they do, then the planetary outflow would be sufficiently great to explain many $\lambda$ Boo abundances.

This model might apply to to HR 8799, a main-sequence A-type star orbited by planets in wide orbits (Marois et al. 2010) which is also is a "mild" $\lambda$ Boo star (Grav \& Kave 1999). Because HR 8799 emits $1.3 \times 10^{28} \mathrm{erg} \mathrm{s}^{-1}$ in the $0.2-2 \mathrm{keV}$ band (Robrade \& Schmitt 2010), a wind from a hot Jupiter - an additional planet interior to those already known - might account for the photospheric abundances.

In this analysis, we assume that hot Jupiters have an atmosphere with nearly a solar composition. Although this result is unestablished (Burrows 2014), at least Na and K have been detected in the outer atmospheres of hot Jupiters (Charbonneau et al. 2002; Sing et al. 2015). Further, winds from hot Jupiters display evidence for Mg (Fossati et al. 
2010; Haswell et al. 2012; Vidal-Madjar et al. 2013) and possibly Si (Linsky et al. 2010; Ballester \& Ben-Jaffel 2015). Current data are consistent with the hypothesis that the abundances in the atmospheres of hot Jupiters are similar to the material accreted on $\lambda$ Boo stars.

\section{DISCUSSION}

Winds from hot Jupiters may be the source of accretion for many $\lambda$ Boo stars. For F,G and $\mathrm{K}$ stars, the frequency of hot Jupiters is $1.2 \% \pm 0.38 \%$ (Wright et al. 2012). Although the frequency of hot Jupiters orbiting main-sequence A-type stars is unknown, the fraction of stars with giant planets appears to peak for stellar masses near $1.9 \mathrm{M}_{\odot}$ (Reffert et al. 2015), and it is plausible that the frequency of hot Jupiters around A-type stars is sufficiently high to explain many $\lambda$ Boo stars.

If the wind from a hot Jupiter provides the material accreted onto a $\lambda$ Boo star, then it may be possible to detect transits or more subtle photometric variations for systems inclined less than $90^{\circ}$. Balona (2013) has analyzed the Kepler light curves for $\sim 2000$ A-type stars and found 166 candidate hot Jupiters (Balona 2014). Further study is required to determine how many of these systems truly harbor planets.

In our proposal, some of the elements in a wind from hot a Jupiter are expelled into the interstellar medium by the star's radiation pressure and therefore mimic a stellar wind. This scenario might explain the observed outflow apparently from Sirius (Bertin et al. 1995) which is not otherwise well understood. Usually the wind speed is comparable to the escape velocity, and the modest blueshift of between $-20 \mathrm{~km} \mathrm{~s}^{-1}$ to $-80 \mathrm{~km} \mathrm{~s}^{-1}$ in the outflow attributed to Sirius may instead be from a planet.

One observational argument against our proposed scenario is that HD 15028 - the host star of WASP 33, an A-type star with a hot Jupiter- is classified as an Am star (Collier-Cameron et al. 2010) and not a $\lambda$ Boo star. However, if the wind from the planet is magnetically funneled onto the host star, then perhaps elemental separations would not occur. It may be that only some winds from hot Jupiters lead to the $\lambda$ Boo pattern of abundances. Another observational argument against our model is that two $\lambda$ Boo stars identified in the Kepler survey (Niemczura et al. 2015) are not obvious candidates for harboring a hot Jupiter (Balona 2014). However, this sample is tiny and the result inconclusive. Despite difficulties, the scenario of accretion from hot Jupiters appears to be the least unlikely of all current proposals to explain $\lambda$ Boo stars.

Future observational tests of $\lambda$ Boo models can be performed with the well defined set 
of stars provided by Murphy et al. (2015).

\section{CONCLUSIONS}

We argue that empirical bounds exclude interstellar matter as the source of accreted material onto $\lambda$ Boo stars. We then propose two modifications to the popular model to explain the abundance pattern in $\lambda$ Boo stars. First, we suggest that radiation pressure on individual gas-phase ions might be an important mechanism for selecting which elements can be accreted. Second, we suggest that the matter acquired by some $\lambda$ Boo stars may originate in the wind from a hot Jupiter.

This work has been partly supported by the NSF. I thank B. Hansen and B. Zuckerman for helpful comments.

\section{REFERENCES}

Anderson, D. R., Smith, A. M. S., Lanotte, A. A. et al. 2011, MNRAS, 416, 2108

Ballester, G. E. \& Ben-Jaffel, L. 2015, 804, 116

Balona, L. A. 2013, MNRAS, 431, 2240

Balona, L. A. 2014, MNRAS, 441, 3543

Bento, J. Wheatley, P. J., Copperwheat, C. M. et al. 2014, MNRAS, 437, 1511

Bergeron, P., Wesemael, F., Dufour, P. et al. 2011, ApJ, 737, 28

Bertin, P., Lamers, H. J. G. L. M., Vidal-Madjar, A., Ferlet, R., \& Lallement, R. 1995, A\&A, 302,899

Bouret, J.-C., Deleuil, M., Lanz, T. et al. 2002, A\&A, 390, 1049

Bourrier, V., Lecalelier des Etangs, A., \& Vidal-Madjar, A. 2015, A\&A, 573, A11

Burrows, A. 2014, Nature, 513, 345

Charbonneau, D., Brown, T. M., Noyes, R. W., \& Gilliland, R. L. 2002, ApJ, 568, 377

Collier-Cameron, A., Guenther, E., Smalley, B. et al. 2010, MNRAS, 407, 507 
Cowley, C. R. 2014, in Putting A Stars into Context: Evolution, Environment, and Related Stars, Eds: G. Mathys et al.; Moscow Publishing House

Cowley, C. R.,Hubrig, S., \& Przybilla, N. 2014, MNRAS, 440, 2457

Debes, J. H. 2006, ApJ, 652, 636

Farihi, J., Barstow, M., A., Redfield, S. et al. 2010, MNRAS, 404, 2123

Fernandez, R., Brandeker, A., \& Wu, Y. 2006, ApJ, 643, 509

Folsom, C.P., Bagnulo, S., Wade, G. A. et al. 2014, MNRAS, 422, 2072

Fossati, L., Haswell, C. A., Froning, C. et al. 2010, ApJ, 714, L222

Gray, R. O., \& Corbally, C. J. 1992, AJ, 124, 989

Gray, R. O., \& Kaye, A. B. 1999, AJ, 118, 2993

Griffin, R. E., Gray, R. O., \& Corbally, C.J. 2012, A\&A, 547, 8

Gunther, H. M., Wolk, S. J., Drake, J. J. et al. 2012, ApJ, 750, 78

Haswell, C. A., Fossatim, L., Ayres, T. et al., 2012, ApJ, 760, 79

Heiter, U. 2002, A\&A, 381, 959

Heiter, U., Weiss, W. W., \& Paunzen, E. 2002, A\&A, 381, 971

Jura, M., Dufour, P., Xu, S. et al. 2015, ApJ, 799, 109

Jura, M. \& Xu, S. 2012, AJ, 143, 6

Jura, M. \& Young, E. D. 2014, Ann. Rev. Earth Planet. Sci., 42, 45

Kamp, I., Hempel, M., Holweger, H. 2002, A\&A, 388, 978

Kamp, I., \& Paunzen, E. 2002, MNRAS, 335, L45

King, J. R. 1994, MNRAS, 269, 209

Kleinman, S. J., Kepler, S. O., Koester, D. et al. 2013, ApJS, 204, 5

Koester, D. 1976, A\&A, 52, 415

Koester, D. 2009, A\&A, 498, 517 
Koester, D., Gaensicke, B., \& Farihi, J. 2014, A\&A, 566, 34

Kurucz, R. L. 1979, ApJS, 40, 1

Lallement, R., Welsh, B. Y., Vergely, J. L., Crifo, F., \& Sfeir, D. 2003, A\&A, 411, 447

Lanz, T., Heap, S. R., \& Hubeny, I. 1995, ApJ, 447, L41

Lanza, A. F. 2013, A\&A, 557, A31

Linsky, J. L., Yang, H., France, K. et al. 2010, ApJ, 717, 1291

Lodders, K. 2003, ApJ, 591, 1220

Marois, C., Zuckerman, B., Konopacky, Q. M., Macintosh, B., \& Barman, T. 2010, Nature, 468,1080

Martinez-Galarza, J. R., Kamp, I., Su, K. Y. L., et al. 2009, ApJ, 694, 165

Morton, D. C. 1991, ApJS, 77, 119

Murphy, S. J., Corbally, C. J., Gray, R. O. et al. 2015, MNRAS, in press, astroph 1508.03633

Murray-Clay, R., Chiang, E. I., \& Murray, N 2009, ApJ, 693, 23

Narusawa, S., Ozaki, S., Kambe, E., \& Sadakane, K. 2006, PASJ, 58, 617

Niemczura, E., Murphy, S. J., Smalley, B. et al. 2015, MNRAS, 450, 2764

Owen, J. E. \& Adams, F. C. 2014, MNRAS, 444, 3761

Paczynski, B. 1971, ARA\&A, 19, 183

Paunzen, E. 1991, A\&A, 373, 633

Paunzen, E., Iliev, I. Kh., Kamp, I. \& Barzova, I. S. 2002, MNRAS, 336, 1030

Paunzen, E., Kamp, I., Weiss, W. W., \& Wiesemeyer, H. 2003, A\&A, 404, 579

Reffert, S., Bergmann, C., Quirrenbach, A., Trifonov, T., \& Kunstler, A. 2015, A\&A, 574, A116

Robrade, J. \& Schmitt, H. H. M. M. 2010, A\&A, 516, A38

Schroder, C. \& Schmitt, J. H. M. M. 2007, A\&A, 475, 677 
Shkolnik, E., Walker, G. A. H., Bohlender D. A., Bu, P.-G., \& Kurster, M. 2005, ApJ, 622, 1075

Simon, T., Ayres, T. R., Redfield, S., \& Linsky, J. 2002, ApJ, 579, 800

Sing, D. K., Wakeford, H. R., Showman, A. P. et al. 2015, MNRAS, 446, 2428

Stutz, Ch., \& Paunzen, E. 2006, A\&A, 458, L17

Turcotte, S. 2002, ApJ, 573, L129

Turcotte, S. \& Charbonneau, P. 1993, ApJ, 413, 376

Venn, K. A., \& Lambert, D. L. 1990, ApJ, 363, 234

Vidal-Madjar, A., Huitson, C. M., Bourrier, V. et al. 2013, A\&A, 560, A54

Xie, J.-W., Brandeker, A., \& Wu, Y. 2013, ApJ, 762, 114

Waters, L. B. F. M., Trams, N. R., \& Waelkens, C. 1992, A\&A, 262, L37

Wilson, P. A., Sing, D. K., Nikolov, N. et al. 2015, MNRAS, 450, 192

Wood, B. E., Muller, H.-R., Zank, G. P, \& Linsky, J. L. 2002, ApJ, 574, 412

Wright, J. T., Marcy, G. W., Howard, A. W. et al. 2012, ApJ, 753, 160

Zuckerman, B. \& Song, I. 2012, ApJ, 758, 77 